\title{
GMM for Panel Count Data Models
}

\author{
Frank Windmeijer
}

Discussion Paper No. 06/591

October 2006

Department of Economics

University of Bristol

8 Woodland Road

Bristol BS8 1TN 


\title{
GMM for Panel Count Data Models*
}

\author{
Frank Windmeijer \\ cemmap \\ and \\ Department of Economics \\ University of Bristol
}

\begin{abstract}
This chapter gives an account of the recent literature on estimating models for panel count data. Specifically, the treatment of unobserved individual heterogeneity that is correlated with the explanatory variables and the presence of explanatory variables that are not strictly exogenous are central. Moment conditions are discussed for these type of problems that enable estimation of the parameters by GMM. As standard Wald tests based on efficient two-step GMM estimation results are known to have poor finite sample behaviour, alternative test procedures that have recently been proposed in the literature are evaluated by means of a Monte Carlo study.
\end{abstract}

JEL Classification: C12, C13, C23

Keywords: GMM, Exponential Models, Hypothesis Testing

*Prepared for Lászlo, M. and P. Sevestre (eds.) The Econometrics of Panel Data. Fundamentals and Recent Developments in Theory, Springer. 


\section{Introduction}

This chapter gives an account of the recent literature on estimating models for panel count data. Specifically, the treatment of unobserved individual heterogeneity that is correlated with the explanatory variables and the presence of explanatory variables that are not strictly exogenous are central. Moment conditions are discussed for these type of problems that enable estimation of the parameters by the Generalised Method of Moments (GMM). Interest in exponential regression models has increased substantially in recent years. The Poisson regression model for modelling an integer count dependent variable is an obvious example where the conditional mean function is routinely modelled to be exponential. But also models for continuous positive dependent variables that have a skewed distribution are increasingly being advocated to have an exponential conditional mean function. Although for these data the log transformation can be applied to multiplicative models, the "retransformation" problem often poses severe difficulties if the object of interest is the level of for example costs, see e.g. Manning, Basu and Mullahy (2005). Santos Silva and Tenreyro (2006) also strongly recommend to estimate the multiplicative models directly, as the log transformation can be unduly restrictive. Although the focus of this chapter is on models for count data, almost all procedures can directly be applied to models where the dependent variable is a positive continuous variable and the conditional mean function is exponential. The one exception is the linear feedback model as described in Section 4, which is a dynamic model specification specific to discrete count data.

Section 2 discusses instrumental variables estimation for count data models in cross sections. Section 3 derives moment conditions for the estimation of models for count panel data allowing for correlated fixed effects and weakly exogenous regressors. Section 4 discusses GMM estimation. Section 5 reviews some of the applied literature and software to estimate the models by nonlinear GMM. As standard Wald tests based on efficient twostep GMM estimation results are known to have poor finite sample behaviour, Section 6 considers alternative test procedures that have recently been proposed in the literature. It 
also considers estimation by the continuous updating estimator (CUE) as this estimator has been shown to have a smaller finite sample bias than one- and two-step GMM. As asymptotic standard errors for the CUE are downward biased in finite samples we use results from alternative, many weak instrument asymptotics (Newey and Windmeijer (2005)) that lead to a larger asymptotic variance of the CUE. The various estimation and test procedures are evaluated by means of a small Monte Carlo study.

\section{GMM in cross-sections}

The Poisson distribution for an integer count variable $y_{i}, i=1, \ldots, N$, with mean $\mu_{i}$ is given by

$$
P\left(y_{i}\right)=\frac{e^{-\mu_{i}} \mu^{y_{i}}}{y_{i} !}
$$

and the Poisson regression model specifies $\mu_{i}=\exp \left(x_{i}^{\prime} \beta\right)$, where $x_{i}$ is a vector of explanatory variables and $\beta$ a parameter vector to be estimated. The log-likelihood function for the sample is then given by

$$
\ln L=\sum_{i=1}^{N} y_{i} \ln \left(\mu_{i}\right)-\mu_{i}-\ln \left(y_{i} !\right)
$$

with first-order condition

$$
\frac{\partial \ln L}{\partial \beta}=\sum_{i=1}^{N} x_{i}\left(y_{i}-\mu_{i}\right)=0 .
$$

It is therefore clear that the Poisson regression estimator is a method of moments estimator. If we write the model with an additive error term $u_{i}$ as

$$
y_{i}=\exp \left(x_{i}^{\prime} \beta\right)+u_{i}=\mu_{i}+u_{i}
$$

with

$$
E\left(x_{i} u_{i}\right)=E\left(x_{i}\left(y_{i}-\mu_{i}\right)\right)=0,
$$

this is clearly the same as the first order condition in the Poisson regression model.

An alternative moment estimator is obtained by specifying the error term as multiplicative in the model

$$
y_{i}=\exp \left(x_{i}^{\prime} \beta\right) \nu_{i}=\mu_{i} w_{i}
$$


with associated moment conditions

$$
E\left(\left(w_{i}-1\right) \mid x_{i}\right)=E\left(\left(\frac{y_{i}-\mu_{i}}{\mu_{i}}\right) \mid x_{i}\right)=0 .
$$

Mullahy (1997) was the first to introduce GMM instrumental variables estimation of count data models with endogenous explanatory variables. He used the multiplicative setup with $x_{i}$ being correlated with the unobservables $w_{i}$ such that $E\left(\left(w_{i}-1\right) \mid x_{i}\right) \neq 0$, and the moment estimator that solves (2) is no longer consistent. There are instruments $z_{i}$ available that are correlated with the endogenous regressors, but not with $w_{i}$ such that

$$
E\left(\left(w_{i}-1\right) \mid z_{i}\right)=E\left(\left(\frac{y_{i}-\mu_{i}}{\mu_{i}}\right) \mid z_{i}\right)=0
$$

Denote $^{1}$

$$
g_{i}=z_{i}\left(\frac{y_{i}-\mu_{i}}{\mu_{i}}\right)
$$

then the GMM estimator for $\beta$ that minimises

$$
Q_{N}(\beta)=\left(\frac{1}{N} \sum_{i=1}^{N} g_{i}\right)^{\prime} W_{N}^{-1}\left(\frac{1}{N} \sum_{i=1}^{N} g_{i}\right)
$$

is consistent, where $W_{N}$ is a weight matrix. The efficient two-step weight matrix is given by

$$
W_{N}\left(\widehat{\beta}_{1}\right)=\frac{1}{N} \sum_{i=1}^{N} g_{i}\left(\widehat{\beta}_{1}\right) g_{i}\left(\widehat{\beta}_{1}\right)^{\prime}
$$

where

$$
g_{i}\left(\widehat{\beta}_{1}\right)=z_{i}\left(\frac{y_{i}-\exp \left(x_{i}^{\prime} \widehat{\beta}_{1}\right)}{\exp \left(x_{i}^{\prime} \widehat{\beta}_{1}\right)}\right)
$$

with $\widehat{\beta}_{1}$ an initial consistent estimator. Angrist (2001) strengthens the arguments for using these moment conditions for causal inference as he shows that in a model with endogenous treatment and a binary instrument, the Mullahy procedure estimates a proportional local average treatment effect (LATE) parameter in models with no covariates.

Windmeijer and Santos Silva (1997) propose use of the additive moment conditions

$$
E\left(\left(y_{i}-\mu_{i}\right) \mid z_{i}\right)=0,
$$

\footnotetext{
${ }^{1}$ From the conditional moments $(3)$ it follows that any function $h(z)$ are valid instruments, which raises the issue of optimal instruments. Here, we will only consider $h(z)=z$.
} 
estimating the parameters $\beta$ again by GMM, with in this case $g_{i}=z_{i}\left(y_{i}-\mu_{i}\right)$. They and Mullahy (1997) compare the two sets of moment conditions and show that both sets cannot in general be valid at the same time. One exception is when there is classical measurement error in an explanatory variable, as in that case both additive and multiplicative moment conditions are valid. Consider the simple model

$$
y_{i}=\exp \left(\alpha+x_{i}^{*} \beta\right)+u_{i}
$$

but $x_{i}^{*}$ is not observed. Instead we observe $x_{i}$

$$
x_{i}=x_{i}^{*}+\varepsilon_{i}
$$

and estimate $\beta$ in the model

$$
y_{i}=\exp \left(\alpha+x_{i} \beta-\varepsilon_{i} \beta\right)+u_{i}
$$

When instruments $z_{i}$ are available that are correlated with $x_{i}$ and independent of the i.i.d measurement errors $\varepsilon_{i}$, then the multiplicative moment conditions

$$
E\left(\left(\frac{y_{i}-\widetilde{\mu}_{i}}{\widetilde{\mu}_{i}}\right) \mid z_{i}\right)=0
$$

are valid, where

$$
\begin{aligned}
\widetilde{\mu}_{i} & =\exp \left(\widetilde{\alpha}+x_{i} \beta\right) \\
\widetilde{\alpha} & =\alpha+\ln (E[\exp (-\varepsilon \beta) \mid z]),
\end{aligned}
$$

and the latter expectation is assumed to be a constant. However, also the additive moment conditions are valid as

$$
E\left(\left(y_{i}-\widetilde{\widetilde{\mu}}_{i}\right) \mid z_{i}\right)=0
$$

where

$$
\begin{aligned}
\widetilde{\widetilde{\mu}}_{i} & =\exp \left(\widetilde{\widetilde{\alpha}}+x_{i} \beta\right) \\
\widetilde{\widetilde{\alpha}} & =\alpha-\ln (E[\exp (-\varepsilon \beta) \mid z]),
\end{aligned}
$$

and so an estimate for $\alpha$ can be recovered as well as the average of the two intercept estimates. 


\section{Panel Data Models}

Let $y_{i t}$ denote the discrete count variable to be explained for subject $i, i=1, \ldots, N$, at time $t, t=1, \ldots, T$; and let $x_{i t}$ denote a vector of explanatory variables. An important feature in panel data applications is unobserved heterogeneity or individual fixed effects. For count data models these effects are generally modelled multiplicatively as

$$
\begin{aligned}
y_{i t} & =\exp \left(x_{i t}^{\prime} \beta+\eta_{i}\right)+u_{i t} \\
& =\mu_{i t} \nu_{i}+u_{i t},
\end{aligned}
$$

where $\nu_{i}=\exp \left(\eta_{i}\right)$ is a permanent scaling factor for the individual specific mean. In general, it is likely that the unobserved heterogeneity components $\eta_{i}$ are correlated with the explanatory variables, $E\left(x_{i t} \eta_{i}\right) \neq 0$, and therefore standard random effects estimators for $\beta$ will be inconsistent, see Hausman, Hall and Griliches (1984). This section will describe moment conditions that can be used to consistently estimate the parameters $\beta$ when there is correlation between $\eta_{i}$ and $x_{i t}$ and allowing for different exogeneity properties of the explanatory variables, i.e. the regressors being strictly exogenous, predetermined or endogenous. Throughout we assume that the $u_{i t}$ are not serially correlated and that $E\left(u_{i t} \mid \nu_{i}\right)=0, t=1, \ldots, T$.

\subsection{Strictly Exogenous Regressors}

When the $x_{i t}$ are strictly exogenous, there is no correlation between any of the idiosyncratic shocks $u_{i s}, s=1, \ldots, T$ and any of the $x_{i t}, t=1, \ldots T$, and the conditional mean of $y_{i t}$ satisfies

$$
E\left(y_{i t} \mid \nu_{i}, x_{i t}\right)=E\left(y_{i t} \mid \nu_{i}, x_{i 1}, \ldots, x_{i T}\right)
$$

For this case, Hausman, Hall and Griliches (1984) use the Poisson conditional maximum likelihood estimator (CMLE), conditioning on $\sum_{t=1}^{T} y_{i t}$, which is the sufficient statistic for $\eta_{i}$. This method mimics the fixed effect logit approach of Chamberlain (1984). However, the Poisson maximum likelihood estimator (MLE) for $\beta$ in a model with separate individual specific constants does not suffer from the incidental parameters problem, and 
is therefore consistent and is the same as the CMLE. To see this, note that the maximum likelihood first order conditions for the $\nu_{i}$ are given by

$$
\frac{\partial \ln L}{\partial \nu_{i}}=\sum_{t=1}^{T} \frac{\partial\left(y_{i t} \ln \left(\mu_{i t} \nu_{i}\right)-\mu_{i t} \nu_{i}\right)}{\partial \nu_{i}}=\sum_{t=1}^{T}\left(\frac{y_{i t}}{\nu_{i}}-\mu_{i t}\right)=0
$$

and therefore the MLE for $\nu_{i}$ is given by

$$
\widehat{\nu}_{i(M L)}=\frac{\bar{y}_{i}}{\bar{\mu}_{i}},
$$

where $\bar{y}_{i}=T^{-1} \sum_{t=1}^{T} y_{i t}$ and $\bar{\mu}_{i}=T^{-1} \sum_{t=1}^{T} \exp \left(x_{i t}^{\prime} \beta\right)$. The MLE of the fixed effect is independent of $\nu_{i}$. Substituting the fixed effects estimates in the first order conditions for $\beta$ results in he moment conditions

$$
\frac{\partial \ln L}{\partial \beta}\left(\widehat{\nu}_{i}\right)=\sum_{i=1}^{N} \sum_{t=1}^{T}\left(y_{i t}-\mu_{i t} \frac{\bar{y}_{i}}{\bar{\mu}_{i}}\right) x_{i t}=0 .
$$

When $x_{i t}$ is strictly exogenous,

$$
\operatorname{plim}_{N \rightarrow \infty} \frac{1}{N} \frac{\partial \ln L}{\partial \beta}\left(\widehat{\nu}_{i}\right)=\operatorname{plim}_{N \rightarrow \infty} \frac{1}{N} \sum_{i=1}^{N} \sum_{t=1}^{T}\left(u_{i t}-\frac{\mu_{i t}}{\bar{\mu}_{i}} \bar{u}_{i}\right) x_{i t}=0,
$$

with $\bar{u}_{i}=T^{-1} \sum_{t=1}^{T} u_{i t}$, and therefore the MLE for $\beta$ is consistent. ${ }^{2}$ It is further identical to the CMLE. The latter can be seen as follows. The Poisson conditional log likelihood function is given by

$$
\ln C L=\sum_{i=1}^{N} \sum_{t=1}^{T} \Gamma\left(y_{i t}+1\right)-\sum_{i=1}^{N} \sum_{t=1}^{T} y_{i t} \ln \left[\sum_{s=1}^{T} \exp \left(-\left(x_{i t}-x_{i s}\right)^{\prime} \beta\right)\right],
$$

where $\Gamma$ (.) is the gamma function, see Hausman, Hall and Griliches (1984, p. 919). The first-order condition for $\beta$ is

$$
\begin{aligned}
\frac{\partial \ln C L}{\partial \beta} & =\sum_{i=1}^{N} \sum_{t=1}^{T} \frac{y_{i t}}{\sum_{s=1}^{T} \exp \left(-\left(x_{i t}-x_{i s}\right)^{\prime} \beta\right)} \sum_{s=1}^{T} \exp \left(-\left(x_{i t}-x_{i s}\right)^{\prime} \beta\right)\left(x_{i t}-x_{i s}\right) \\
& =\sum_{i=1}^{N} \sum_{t=1}^{T} y_{i t} x_{i t}-\sum_{i=1}^{N} \sum_{t=1}^{T} y_{i t} \frac{\sum_{s=1}^{T} x_{i s} \exp \left(x_{i s}^{\prime} \beta\right)}{\sum_{s=1}^{T} \exp \left(x_{i s}^{\prime} \beta\right)} \\
& =\sum_{i=1}^{N} \sum_{t=1}^{T} x_{i t}\left(y_{i t}-\mu_{i t} \frac{\bar{y}_{i}}{\bar{\mu}_{i}}\right),
\end{aligned}
$$

\footnotetext{
${ }^{2}$ Lancaster (2002) finds the same result for the Poisson model by means of a decomposition of the likelihood.
} 
which is exactly the same as the MLE first order condition for $\beta$.

The first order conditions imply that the Poisson MLE for $\beta$ is equivalent to the moment estimator in a model where the ratio of individual, or within group, means are used to approximate the individual specific effects. This mean scaling model is given by

$$
y_{i t}=\mu_{i t} \frac{\bar{y}_{i}}{\bar{\mu}_{i}}+u_{i t}^{*},
$$

where $u_{i t}^{*}=u_{i t}-\frac{\mu_{i t}}{\bar{\mu}_{i}} \bar{u}_{i}$. Blundell, Griffith and Windmeijer (2002) call the resulting estimator the within group mean scaling estimator. ${ }^{3}$

\subsection{Predetermined Regressors}

A regressor is predetermined when it is not correlated with current and future shocks, but it is correlated with past shocks:

$$
\begin{aligned}
& E\left(x_{i t} u_{i t+j}\right)=0, \quad j \geq 0 \\
& E\left(x_{i t} u_{i t-s}\right) \neq 0, \quad s \geq 1 .
\end{aligned}
$$

With predetermined regressors, the within group mean scaling estimator is no longer consistent. Chamberlain (1992) has proposed transformations that eliminate the fixed effect from the multiplicative model and generate orthogonality conditions that can be used for consistent estimation in count data models with predetermined regressors. The quasi-differencing transformation is

$$
s_{i t}=y_{i t} \frac{\mu_{i t-1}}{\mu_{i t}}-y_{i t-1}=u_{i t} \frac{\mu_{i t-1}}{\mu_{i t}}-u_{i t-1} .
$$

Let $x_{i}^{t-1}=\left(x_{i 1}, \ldots, x_{i t-1}\right)$. When $x_{i t}$ is predetermined, the following moment conditions hold:

$$
E\left(s_{i t} \mid x_{i}^{t-1}\right)=E\left(\left(E\left(u_{i t} \mid x_{i}^{t}\right) \frac{\mu_{i t-1}}{\mu_{i t}}-u_{i t-1}\right) \mid x_{i}^{t-1}\right)=0 .
$$

Wooldridge (1991) proposed the following quasi-differencing transformation

$$
q_{i t}=\frac{y_{i t}}{\mu_{i t}}-\frac{y_{i t-1}}{\mu_{i t-1}}=\frac{u_{i t}}{\mu_{i t}}-\frac{u_{i t-1}}{\mu_{i t-1}},
$$

\footnotetext{
${ }^{3}$ Clearly, the Poisson pseudo-likelihood results are preserved, see also Wooldridge (1999).
} 
with moment conditions

$$
E\left(q_{i t} \mid x_{i}^{t-1}\right)=E\left(\left(\frac{E\left(u_{i t} \mid x_{i}^{t}\right)}{\mu_{i t}}-\frac{u_{i t-1}}{\mu_{i t-1}}\right) \mid x_{i}^{t-1}\right)=0 .
$$

It is clear that a variable in $x_{i t}$ can not have only non-positive or non-negative values, as then the corresponding estimate for $\beta$ is infinity. A way around this problem is to transform $x_{i t}$ in deviations from its overall mean, $\widetilde{x}_{i t}=x_{i t}-\bar{x}$, with $\bar{x}=\frac{1}{N T} \sum_{i=1}^{N} \sum_{t=1}^{T} x_{i t}$, see Windmeijer (2000).

Both moment conditions can also be derived from a multiplicative model specification

$$
y_{i t}=\exp \left(x_{i t}^{\prime} \beta+\eta_{i}\right) w_{i t}=\mu_{i t} \nu_{i} w_{i t},
$$

where $x_{i t}$ is now predetermined w.r.t. $w_{i t}$. Again, we assume that the $w_{i t}$ are not serially correlated and not correlated with $\nu_{i}$, and $E\left(w_{i t}\right)=1$. The Chamberlain quasidifferencing transformation in this case is equivalent to

$$
s_{i t}=y_{i t} \frac{\mu_{i t-1}}{\mu_{i t}}-y_{i t-1}=\nu_{i} \mu_{i t-1}\left(w_{i t}-w_{i t-1}\right),
$$

with moment conditions

$$
E\left(s_{i t} \mid x_{i}^{t-1}\right)=E\left(\nu_{i} \mu_{i t-1} E\left(\left(w_{i t}-w_{i t-1}\right) \mid \nu_{i}, x_{i}^{t-1}\right) \mid x_{i}^{t-1}\right)=0 .
$$

Equivalently, for the Wooldridge transformation,

$$
q_{i t}=\frac{y_{i t}}{\mu_{i t}}-\frac{y_{i t-1}}{\mu_{i t-1}}=\nu_{i}\left(w_{i t}-w_{i t-1}\right)
$$

and

$$
E\left(q_{i t} \mid x_{i}^{t-1}\right)=E\left(\nu_{i} E\left(\left(w_{i t}-w_{i t-1}\right) \mid \nu_{i}, x_{i}^{t-1}\right) \mid x_{i}^{t-1}\right)=0
$$

\subsection{Endogenous Regressors}

Regressors are endogenous when they are correlated with current (and possibly past) shocks $E\left(x_{i t} u_{i t-s}\right) \neq 0, s \geq 0$, for the specification with additive errors $u_{i t}$, or when $E\left(x_{i t} w_{i t-s}\right) \neq 0, s \geq 0$, for the specification with multiplicative errors $w_{i t}$. It is clear from the derivations in the previous section that we cannot find valid sequential conditional 
moment conditions for the specification with additive errors due to the non-separability of the $u_{i t}$ and $\mu_{i t}$. For the multiplicative error specification, there is again non-separability of $\mu_{i t-1}$ and $\left(w_{i t}-w_{i t-1}\right)$ for the Chamberlain transformation and so

$$
E\left(s_{i t} \mid x_{i}^{t-2}\right)=E\left(\nu_{i} \mu_{i t-1} E\left(\left(w_{i t}-w_{i t-1}\right) \mid \nu_{i}, x_{i}^{t-1}\right) \mid x_{i}^{t-2}\right) \neq 0
$$

In contrast, the Wooldridge transformation does not depend on $\mu_{i t}$ or $\mu_{i t-1}$ in this case. Valid moment conditions are then given by

$$
E\left(q_{i t} \mid x_{i}^{t-2}\right)=E\left(\nu_{i} E\left(\left(w_{i t}-w_{i t-1}\right) \mid \nu_{i}, x_{i}^{t-2}\right) \mid x_{i}^{t-2}\right)=0 .
$$

Therefore, in the case of endogenous explanatory variables, only the Wooldridge transformation can be used for the consistent estimation of the parameters $\beta$. This includes the case of classical measurement error in $x_{i t}$, where the measurement error is not correlated over time.

\subsection{Dynamic Models}

Specifying dynamic models for count data by including lags of the dependent count variables in the explanatory part of the model is not as straightforward as with linear models for a continuous dependent variable. Inclusion of the lagged dependent variable in the exponential mean function may lead to rapidly exploding series. A better starting point is to specify the model as in Crépon and Duguet (1997)

$$
y_{i t}=h\left(y_{i t-1}, \gamma\right) \exp \left(x_{i t}^{\prime} \beta+\eta_{i}\right)+u_{i t}
$$

where $h(.,)>$.0 is any given function describing the way past values of the dependent variable are affecting the current value.

Let

$$
d_{i t}=1_{\left\{y_{i t}=0\right\}}
$$

then a possible choice for $h(.,$.$) is$

$$
h\left(y_{i t}, \gamma\right)=\exp \left(\gamma_{1} \ln \left(y_{i t-1}+c d_{i t-1}\right)+\gamma_{2} d_{i t-1}\right)
$$


where $c$ is a pre-specified constant. In this case, $\ln \left(y_{i t-1}\right)$ is included as a regressor for positive $y_{i t-1}$, and zero values of $y_{i t-1}$ have a separate effect on current values of $y_{i t}$. Crépon and Duguet (1997) considered

$$
h\left(y_{i t}, \gamma\right)=\exp \left(\gamma\left(1-d_{i t-1}\right)\right)
$$

and extensions thereof to several regime indicators.

Blundell, Griffith and Windmeijer (2002) propose use of a linear feedback model for modelling dynamic count panel data process. The linear feedback model of order 1 $(\operatorname{LFM}(1))$ is defined as

$$
\begin{aligned}
y_{i t} & =\gamma y_{i t-1}+\exp \left(x_{i t}^{\prime} \beta+\eta_{i}\right)+u_{i t} \\
& =\gamma y_{i t-1}+\mu_{i t} \nu_{i}+u_{i t}
\end{aligned}
$$

where the lag of the dependent variable enters the model linearly. Extensions to include further lags are straightforward. The LFM has its origins in the Integer-Valued Autoregressive (INAR) process and can be motivated as an entry-exit process with the probability of exit equal to $(1-\gamma)$. The correlation over time for the INAR(1) process without additional regressors is similar to that of the $\operatorname{AR}(1)$ model, $\operatorname{corr}\left(y_{i t}, y_{i t-j}\right)=\gamma^{j}$.

For the patents-R\&D model, Blundell, Griffith and Windmeijer (2002) consider the economic model

$$
P_{i t}=k\left(R_{i t}^{\beta}+(1-\delta) R_{i t-1}^{\beta}+(1-\delta)^{2} R_{i t-2}^{\beta} \ldots\right) \nu_{i}+\varepsilon_{i t}
$$

where $P_{i t}$ and $R_{i t}$ are the number of patents and R\&D expenditures for firm $i$ at time $t$ respectively, $k$ is a positive constant and $\mathrm{R} \& \mathrm{D}$ expenditures depreciate geometrically at rate $\delta$. The long run steady state for firm $i$, ignoring feedback from patents to R\&D, can be written as

$$
P_{i}=\frac{k}{\delta} R_{i}^{\beta} \nu_{i}
$$

and $\beta$ can therefore be interpreted as the long run elasticity. Inverting (7) leads to

$$
P_{i t}=k R_{i}^{\beta} \nu_{i}+(1-\delta) P_{i t-1}+u_{i t}
$$


and so in the LFM model

$$
P_{i t}=\gamma P_{i t-1}+\exp \left(k^{*}+\beta \ln \left(R_{i t}\right)\right) \nu_{i}+u_{i t},
$$

the estimate for $\gamma$ is an estimate of the depreciation factor $(1-\delta)$ and the estimate for $\beta$ is an estimate of the long run elasticity of the $\mathrm{R} \& \mathrm{D}$ returns to patents.

Even when the $x_{i t}$ are strictly exogenous, the within group mean scaling estimator will be inconsistent for small $T$, as the lagged dependent variable is a predetermined variable. For estimation by GMM, the Chamberlain quasi-differencing transformation for the $\operatorname{LFM}(1)$ model is given by

$$
s_{i t}=\left(y_{i t}-\gamma y_{i t-1}\right) \frac{\mu_{i t-1}}{\mu_{i t}}-\left(y_{i t-1}-\gamma y_{i t-2}\right)
$$

and the Wooldridge quasi-differencing transformation is given by

$$
q_{i t}=\frac{y_{i t}-\gamma y_{i t-1}}{\mu_{i t}}-\frac{y_{i t-1}-\gamma y_{i t-2}}{\mu_{i t-1}} .
$$

For predetermined $x_{i t}$ the following moment conditions hold

$$
\begin{aligned}
& E\left(s_{i t} \mid y_{i}^{t-2}, x_{i}^{t-1}\right)=0 \\
& E\left(q_{i t} \mid y_{i}^{t-2}, x_{i}^{t-1}\right)=0
\end{aligned}
$$

while for endogenous $x_{i t}$, only the Wooldridge moment conditions are valid

$$
E\left(q_{i t} \mid y_{i}^{t-2}, x_{i}^{t-2}\right)=0
$$

\section{GMM}

The orthogonality conditions as described in the sections above can be used to consistently estimate the model parameters by the GMM estimation technique (see Hansen, 1982). Let $\theta$ be the $k$-vector of parameters to be estimated, e.g. for the LFM(1) model $\theta=\left(\gamma, \beta^{\prime}\right)^{\prime}$. The model has a true parameter $\theta_{0}$ satisfying the $q$ moment conditions

$$
E\left[g_{i}\left(\theta_{0}\right)\right]=0 .
$$


The GMM estimator $\widehat{\theta}$ for $\theta_{0}$ is defined as

$$
\widehat{\theta}=\arg \min _{\theta \in \Theta}\left[\frac{1}{N} \sum_{i=1}^{N} g_{i}(\theta)\right]^{\prime} W_{N}^{-1}\left[\frac{1}{N} \sum_{i=1}^{N} g_{i}(\theta)\right],
$$

where $\Theta$ is a compact set of parameter values; $W_{N}$ satisfies $\operatorname{plim}_{N \rightarrow \infty} W_{N}=W$, with $W$ a positive definite matrix. Regularity conditions are assumed such that $\lim _{N \rightarrow \infty} \frac{1}{N} \sum_{i=1}^{N} g_{i}(\theta)=$ $E\left[g_{i}(\theta)\right]$ and $\frac{1}{\sqrt{N}} \sum_{i=1}^{N} g_{i}\left(\theta_{0}\right) \rightarrow N(0, \Psi)$ where $\Psi=\lim _{N \rightarrow \infty} \frac{1}{N} \sum_{i=1}^{N} E\left[\left(g_{i}\left(\theta_{0}\right) g_{i}\left(\theta_{0}\right)^{\prime}\right)\right]$. Let $\Gamma(\theta)=E\left[\partial g_{i}(\theta) / \partial \theta^{\prime}\right]$ and $\Gamma_{\theta_{0}} \equiv \Gamma\left(\theta_{0}\right)$, then $\sqrt{N}\left(\widehat{\theta}-\theta_{0}\right)$ has a limiting normal distribution,

$$
\sqrt{N}\left(\widehat{\theta}-\theta_{0}\right) \rightarrow N\left(0, V_{W}\right)
$$

where

$$
V_{W}=\left(\Gamma_{\theta_{0}}^{\prime} W^{-1} \Gamma_{\theta_{0}}\right)^{-1} \Gamma_{\theta_{0}}^{\prime} W^{-1} \Psi W^{-1} \Gamma_{\theta_{0}}\left(\Gamma_{\theta_{0}}^{\prime} W^{-1} \Gamma_{\theta_{0}}\right)^{-1}
$$

The efficient two-step GMM estimator, denoted $\widehat{\theta}_{2}$, is based on a weight matrix that satisfies $\operatorname{plim}_{N \rightarrow \infty} W_{N}=\Psi$, with $V_{W}=\left(\Gamma_{\theta_{0}}^{\prime} \Psi^{-1} \Gamma_{\theta_{0}}\right)^{-1}$ in that case. A weight matrix that satisfies this property is given by

$$
W_{N}\left(\widehat{\theta}_{1}\right)=\frac{1}{N} \sum_{i=1}^{N} g_{i}\left(\widehat{\theta}_{1}\right) g_{i}\left(\widehat{\theta}_{1}\right)^{\prime},
$$

where $\widehat{\theta}_{1}$ is an initial consistent estimator for $\theta_{0}$.

Denote $\bar{g}(\theta)=\frac{1}{N} \sum_{i=1}^{N} g_{i}(\theta)$. The standard test for overidentifying restrictions is $N$ times the minimised GMM criterion

$$
N \bar{g}\left(\widehat{\theta}_{2}\right)^{\prime} W_{N}^{-1}\left(\widehat{\theta}_{1}\right) \bar{g}\left(\widehat{\theta}_{2}\right)
$$

which has an asymptotic chi-squared distribution with $q-k$ degrees of freedom when the moment conditions are valid.

For the Chamberlain quasi-differencing transformation the GMM estimator $\widehat{\theta}$ minimises

$$
\widehat{\theta}=\arg \min _{\theta \in \Theta}\left(\frac{1}{N} \sum_{i=1}^{N} s_{i}(\theta)^{\prime} Z_{i}\right) W_{N}^{-1}\left(\frac{1}{N} \sum_{i=1}^{N} Z_{i}^{\prime} s_{i}(\theta)\right),
$$

where, for the $\operatorname{LFM}(1)$ model, $s_{i}(\theta)$ is the $T-2$ vector $\left(s_{i 3}, s_{i 4}, \ldots, s_{i T}\right)^{\prime}$, with $s_{i t}$ as defined in (8), $Z_{i}$ is the matrix of instruments and $W_{N}$ is a weight matrix. When the full 
sequential set of instruments is used and $x_{i t}$ is predetermined, the instrument matrix for the $\operatorname{LFM}(1)$ model is given by

$$
Z_{i}=\left[\begin{array}{llllllllll}
y_{i 1} & x_{i 1} & x_{i 2} & & & & & & & \\
& & & \ddots & & & & & & \\
& & & & y_{i 1} & \cdots & y_{i T-2} & x_{i 1} & \cdots & x_{i T-1}
\end{array}\right] .
$$

The efficient weight matrix is

$$
W_{N}\left(\widehat{\theta}_{1}\right)=\frac{1}{N} \sum_{i=1}^{N} Z_{i}^{\prime} s_{i}\left(\widehat{\theta}_{1}\right) s_{i}\left(\widehat{\theta}_{1}\right)^{\prime} Z_{i}
$$

where $\widehat{\theta}_{1}$ can be a GMM estimator using for example $W_{N}=\frac{1}{N} \sum_{i=1}^{N} Z_{i}^{\prime} Z_{i}$ as the initial weight matrix. As stated above, under the assumed regularity conditions both $\widehat{\theta}_{1}$ and $\widehat{\theta}_{2}$ are asymptotically normally distributed. The asymptotic variance of $\widehat{\theta}_{1}$ is estimated by

$$
\begin{aligned}
\widehat{\operatorname{var}}\left(\widehat{\theta}_{1}\right)= & \frac{1}{N}\left(C\left(\widehat{\theta}_{1}\right)^{\prime} W_{N}^{-1} C\left(\widehat{\theta}_{1}\right)\right)^{-1} C\left(\widehat{\theta}_{1}\right)^{\prime} W_{N}^{-1} W_{N}\left(\widehat{\theta}_{1}\right) W_{N}^{-1} C\left(\widehat{\theta}_{1}\right) \\
& \times\left(C\left(\widehat{\theta}_{1}\right)^{\prime} W_{N}^{-1} C\left(\widehat{\theta}_{1}\right)\right)^{-1}
\end{aligned}
$$

where

$$
C\left(\widehat{\theta}_{1}\right)=\left.\frac{1}{N} \sum_{i=1}^{N} \frac{\partial Z_{i}^{\prime} s_{i}(\theta)}{\partial \theta}\right|_{\widehat{\theta}_{1}} .
$$

The asymptotic variance of the efficient two-step GMM, estimator is estimated by

$$
\widehat{\operatorname{var}}\left(\widehat{\theta}_{2}\right)=\frac{1}{N}\left(C\left(\widehat{\theta}_{2}\right)^{\prime} W_{N}^{-1}\left(\widehat{\theta}_{1}\right) C\left(\widehat{\theta}_{2}\right)\right)^{-1}
$$

\section{Applications and software}

The instrumental variables methods for count data models with endogenous regressors using cross section data, as described in Section 2, have often been applied in the health econometrics literature. For example, Mullahy (1997) uses the multiplicative moment conditions to estimate cigarette demand functions with a habit stock measure as endogenous regressor. Windmeijer and Santos Silva (1997) estimate health care demand functions with a self reported health measure as possible endogenous variable, while VeraHernandez (1999) and Schellhorn (2001) estimate health care demand functions with 
endogenous insurance choice. An example outside the health econometrics literature is Kelly (2000) who models the number of crimes with police activity as an endogenous regressor.

The count panel data literature has largely focused on estimating models for patenting and the returns to R\&D investments, which started with the seminal paper of Hausman, Hall and Griliches (1984). Following the development of the quasi-differencing approach of Chamberlain (1992) and Wooldridge (1991,1997), Montalvo (1997), Cincera (1997), Crépon and Duguet (1997), Blundell, Griffith and Van Reenen (1999) and Blundell, Griffith and Windmeijer (2002) developed and/or estimated patent (or innovation) production functions using the GMM framework allowing for correlated firm specific effects and weakly exogenous inputs. More recently, Kim and Marschke (2005) use the GMM framework to find a relationship between a firms' patenting behaviour and scientist turnover, whereas Salomon and Shaver (2005) estimate a linear feedback model and find that exporting has a positive effect on innovating behaviour of the firm.

The latter two publications estimated the models using ExpEnd, Windmeijer (2002). This is a user friendly open source GAUSS (Aptech Systems, 2005) code for the nonlinear GMM estimation of the models described in the previous sections. ${ }^{4}$ For cross-section data, ExpEnd estimates simple robust Poisson regression models using moment conditions (1); and instrumental variables regressions using Mullahy's (1997) multiplicative moment conditions (3) or the additive moment conditions (4). For panel data, ExpEnd estimates pooled robust Poisson regression models; fixed effects models, using the mean scaling model (5); and the quasi-differencing models using the Chamberlain (1992) or the Wooldridge $(1991,1997)$ transformation, both for static and linear feedback models. For the quasi-differencing models, sequential and so-called stacked IV type instruments can be specified, in both cases allowing for a flexible lag length of the instruments. For overidentified models one- and two-step GMM parameter estimates are reported, together with asymptotic standard errors. The test for overidentifying restrictions is reported and for the panel data models the output further includes a test of first and second order

\footnotetext{
${ }^{4}$ For a review, see Romeu (2004).
} 
serial correlation of the quasi-differencing "residuals" $s_{i t}(\widehat{\theta})$ or $q_{i t}(\widehat{\theta})$. If the model is correctly specified one expects to find an $\mathrm{MA}(1)$ serial correlation structure.

Another package that enables researchers to estimate these model types is TSP, Hall and Cummins (2005). Kitazawa (2000) provides various TSP procedures for the estimation of count panel data models. Also LIMDEP, Greene (2005), provides an environment where these models can be estimated.

\section{Finite sample inference}

Standard Wald tests based on two-step efficient GMM estimators are known to have poor finite sample properties (see e.g. Blundell and Bond (1998)). Bond and Windmeijer (2005) therefore analysed the finite sample performance of various alternative test procedures for testing linear restrictions in linear panel data models. The statistics they found to perform well in Monte Carlo exercises were an alternative two-step Wald test that uses a finite sample correction for the asymptotic variance matrix, the LM test, and a simple criterion-based test. In this section we briefly describe these procedures and adapt them to the case of nonlinear GMM estimation where necessary.

Newey and Smith (2004) have shown that the GMM estimator can further also suffer from quite large finite sample biases and advocate use of Generalized Empirical Likelihood (GEL) estimators that they show to have smaller finite sample biases. We will consider here the performance of the Continuous Updating Estimator (CUE) as proposed by Hansen, Heaton and Yaron (1996), which is a GEL estimator. The Wald test based on the CUE has also been shown to perform poorly in finite samples by e.g. Hansen, Heaton and Yaron (1996). Newey and Windmeijer (2005) derive the asymptotic distribution of the CUE when there are many weak moment conditions. The asymptotic variance in this case is larger than the usual asymptotic one and we will analyse the performance of an alternative Wald test that uses an estimate for this larger asymptotic variance, together with a criterion based test for the CUE as proposed by Hansen, Heaton and Yaron (1996). 
The estimators and test procedures will be evaluated in a Monte Carlo study of testing linear restrictions in a static count panel data model with an explanatory variable that is correlated with the fixed unobserved heterogeneity and which is predetermined. The Chamberlain quasi-differencing transformation will be used with sequential moment conditions.

\subsection{Wald test and finite sample variance correction}

The standard Wald test for testing $r$ linear restrictions of the form $r\left(\theta_{0}\right)=0$ is calculated as

$$
\text { Wald }=r(\widehat{\theta})^{\prime}\left(R^{\prime} \widehat{\operatorname{var}}(\widehat{\theta}) R\right)^{-1} r(\widehat{\theta})
$$

where $R=\partial r(\theta) / \partial \theta^{\prime}$, and has an asymptotic $\chi_{r}^{2}$ distribution under the null. Based on the two-step GMM estimator and using its conventional asymptotic variance estimate, the Wald test has often been found to overreject correct null hypotheses severely compared to its nominal size. This can occur even when the estimator has negligible finite sample bias, due to the fact that the estimated asymptotic standard errors can be severely downward biased in small samples. Windmeijer (2005) proposed a finite sample variance correction that takes account of the extra variation due to the presence of the estimated parameters $\widehat{\theta}_{1}$ in the weight matrix. He showed by means of a Monte Carlo study that this correction works well in linear models, but it is not clear how well it will work in nonlinear GMM.

To derive the finite sample corrected variance, let

$$
\bar{g}(\theta)=\frac{1}{N} \sum_{i=1}^{N} g_{i}(\theta) ; \quad C(\theta)=\frac{\partial \bar{g}(\theta)}{\partial \theta^{\prime}} ; \quad G(\theta)=\frac{\partial C(\theta)}{\partial \theta},
$$

and

$$
\begin{aligned}
b_{\theta_{0}, W_{N}} & =\left.\frac{1}{2} \frac{\partial Q_{W_{N}}}{\partial \theta}\right|_{\theta_{0}}=C\left(\theta_{0}\right)^{\prime} W_{N}^{-1} \bar{g}\left(\theta_{0}\right) \\
A_{\theta_{0}, W_{N}} & =\left.\frac{1}{2} \frac{\partial^{2} Q_{W_{N}}}{\partial \theta \partial \theta^{\prime}}\right|_{\theta_{0}}=C\left(\theta_{0}\right)^{\prime} W_{N}^{-1} C\left(\theta_{0}\right)+G\left(\theta_{0}\right)^{\prime}\left(I_{k} \otimes W_{N}^{-1} \bar{g}\left(\theta_{0}\right)\right) .
\end{aligned}
$$

A standard first order Taylor series approximation of $\widehat{\theta}_{2}$ around $\theta_{0}$, conditional on $W_{N}\left(\widehat{\theta}_{1}\right)$, results in

$$
\widehat{\theta}_{2}-\theta_{0}=-A_{\theta_{0}, W_{N}\left(\widehat{\theta}_{1}\right)}^{-1} b_{\theta_{0}, W_{N}\left(\widehat{\theta}_{1}\right)}+O_{p}\left(N^{-1}\right) .
$$


A further expansion of $\widehat{\theta}_{1}$ around $\theta_{0}$ results in

$$
\widehat{\theta}_{2}-\theta_{0}=-A_{\theta_{0}, W_{N}\left(\theta_{0}\right)}^{-1} b_{\theta_{0}, W_{N}\left(\theta_{0}\right)}+D_{\theta_{0}, W_{N}\left(\theta_{0}\right)}\left(\widehat{\theta}_{1}-\theta_{0}\right)+O_{p}\left(N^{-1}\right)
$$

where

$$
W_{N}\left(\theta_{0}\right)=\frac{1}{N} \sum_{i=1}^{N} g_{i}\left(\theta_{0}\right) g_{i}\left(\theta_{0}\right)^{\prime}
$$

and

$$
D_{\theta_{0}, W_{N}\left(\theta_{0}\right)}=\left.\frac{\partial}{\partial \theta^{\prime}}\left(-A_{\theta_{0}, W_{N}(\theta)}^{-1} b_{\theta_{0}, W_{N}(\theta)}\right)\right|_{\theta_{0}}
$$

is a $k \times k$ matrix.

Let $\widehat{\theta}_{1}$ be a one-step GMM estimator that uses a weight matrix $W_{N}$ that does not depend on estimated parameters. An estimate of the variance of $\widehat{\theta}_{2}$ that incorporates the term involving the one-step parameter estimates used in the weight matrix can then be obtained as

$$
\begin{aligned}
\widehat{\operatorname{var}}_{c}\left(\widehat{\theta}_{2}\right)= & \frac{1}{N} A_{\widehat{\theta}_{2}, W_{N}\left(\widehat{\theta}_{1}\right)}^{-1} C\left(\widehat{\theta}_{2}\right)^{\prime} W_{N}^{-1}\left(\widehat{\theta}_{1}\right) C\left(\widehat{\theta}_{2}\right) A_{\widehat{\theta}_{2}, W_{N}\left(\widehat{\theta}_{1}\right)}^{-1} \\
& +\frac{1}{N} D_{\widehat{\theta}_{2}, W_{N}\left(\widehat{\theta}_{1}\right)} A_{\widehat{\theta}_{1}, W_{N}}^{-1} C\left(\widehat{\theta}_{1}\right)^{\prime} W_{N}^{-1} C\left(\widehat{\theta}_{2}\right) A_{\widehat{\theta}_{2}, W_{N}\left(\widehat{\theta}_{1}\right)}^{-1} \\
& +\frac{1}{N} A_{\widehat{\theta}_{2}, W_{N}}^{-1}\left(\widehat{\theta}_{1}\right) \\
& C\left(\widehat{\theta}_{2}\right)^{\prime} W_{N}^{-1} C\left(\widehat{\theta}_{1}\right) A_{\widehat{\theta}_{1}, W_{N}}^{-1} D_{\widehat{\theta}_{2}, W_{N}\left(\widehat{\theta}_{1}\right)}^{\prime} \\
& +D_{\widehat{\theta}_{2}, W_{N}\left(\widehat{\theta}_{1}\right)} \widehat{\operatorname{var}}\left(\widehat{\theta}_{1}\right) D_{\widehat{\theta}_{2}, W_{N}\left(\widehat{\theta}_{1}\right)}^{\prime},
\end{aligned}
$$

where the $j^{\text {th }}$ column of $D_{\widehat{\theta}_{2}, W_{N}\left(\widehat{\theta}_{1}\right)}$ is given by

$$
D_{\widehat{\theta}_{2}, W_{N}\left(\widehat{\theta}_{1}\right)}[., j]=\left.A_{\widehat{\theta}_{2}, W_{N}\left(\widehat{\theta}_{1}\right)}^{-1} C\left(\widehat{\theta}_{2}\right)^{\prime} W_{N}^{-1}\left(\widehat{\theta}_{1}\right) \frac{\partial W_{N}(\theta)}{\partial \theta_{j}}\right|_{\widehat{\theta}_{2}} W_{N}^{-1}\left(\widehat{\theta}_{1}\right) \bar{g}\left(\widehat{\theta}_{2}\right)
$$

and

$$
\frac{\partial W_{N}(\theta)}{\partial \theta_{j}}=\frac{1}{N} \sum_{i=1}^{N}\left(\frac{\partial g_{i}(\theta)}{\partial \theta_{j}} g_{i}(\theta)^{\prime}+g_{i}(\theta) \frac{\partial g_{i}(\theta)^{\prime}}{\partial \theta_{j}}\right) .
$$

The alternative two-step Wald test that uses a finite sample correction for the asymptotic variance matrix is then defined as

$$
\operatorname{Wald}_{c}=r\left(\widehat{\theta}_{2}\right)^{\prime}\left(R^{\prime} \widehat{\operatorname{var}}_{c}\left(\widehat{\theta}_{2}\right) R\right)^{-1} r\left(\widehat{\theta}_{2}\right)
$$


The term $D_{\theta_{0}, W\left(\theta_{0}\right)}\left(\widehat{\theta}_{1}-\theta_{0}\right)$ in $(9)$ is itself $O_{p}\left(N^{-1}\right)$ and in this general setting, incorporating non-linear models and/or non-linear moment conditions, whether taking account of it will improve the estimation of the small sample variance substantially depends on the other remainder terms which are of the same order.

\subsection{Criterion-Based Tests}

Using the notation as in Bond and Windmeijer (2005), the standard two-step Wald test can be computed as a criterion difference

$$
\text { Wald }=N\left(\bar{g}\left(\widetilde{\theta}_{\widehat{2}}\right)^{\prime} W_{N}^{-1}\left(\widehat{\theta}_{1}\right) \bar{g}\left(\widetilde{\theta}_{\widehat{2}}\right)-\bar{g}\left(\widehat{\theta}_{2}\right)^{\prime} W_{N}^{-1}\left(\widehat{\theta}_{1}\right) \bar{g}\left(\widehat{\theta}_{2}\right)\right),
$$

where $\widehat{\theta}_{1}$ and $\widehat{\theta}_{2}$ are the one-step and two-step GMM estimators in the unrestricted model, whereas $\widetilde{\theta}_{\widehat{2}}$ is a two-step GMM estimator in the restricted model, but using as a weight matrix the consistent estimate of $\Psi$ based on the unrestricted one-step GMM estimator, $W_{N}\left(\widehat{\theta}_{1}\right)$, see Newey and West (1987).

The LM test can also be computed as a criterion difference

$$
\mathrm{LM}=N\left(\bar{g}\left(\widetilde{\theta}_{2}\right)^{\prime} W_{N}^{-1}\left(\widetilde{\theta}_{1}\right) \bar{g}\left(\widetilde{\theta}_{2}\right)-\bar{g}\left(\widehat{\theta}_{\widetilde{2}}\right)^{\prime} W_{N}^{-1}\left(\widetilde{\theta}_{1}\right) \bar{g}\left(\widehat{\theta}_{\tilde{2}}\right)\right)
$$

where $\widetilde{\theta}_{1}$ and $\widetilde{\theta}_{2}$ are the one-step and two-step GMM estimators in the restricted model, whereas $\widehat{\theta}_{\widetilde{2}}$ is a two-step GMM estimator in the unrestricted model, but using as a weight matrix the consistent estimate of $\Psi$ under the null, based on the restricted one-step GMM estimator, $W_{N}\left(\widetilde{\theta}_{1}\right)$. The LM test has an asymptotic $\chi_{r}^{2}$ distribution under the null.

The criterion-based test statistic considered by Bond, Bowsher and Windmeijer (2001) is given by

$$
D_{R U}=N\left(\bar{g}\left(\widetilde{\theta}_{2}\right)^{\prime} W_{N}^{-1}\left(\widetilde{\theta}_{1}\right) \bar{g}\left(\widetilde{\theta}_{2}\right)-\bar{g}\left(\widehat{\theta}_{2}\right)^{\prime} W_{N}^{-1}\left(\widehat{\theta}_{1}\right) \bar{g}\left(\widehat{\theta}_{2}\right)\right)
$$

$D_{R U}$ is the "likelihood ratio" test equivalent for GMM, and is the difference between the test statistics for overidentifying restrictions in the restricted and unrestricted models. Under the null, $D_{R U}$ has an asymptotic $\chi_{r}^{2}$ distribution. 


\subsection{Continuous Updating Estimator}

The Continuous Updating Estimator (CUE) is given by

$$
\begin{aligned}
\widehat{\theta}_{C U} & =\arg \min _{\theta \in \Theta} Q(\theta) \\
Q(\theta) & =\frac{1}{2} \bar{g}(\theta)^{\prime} W_{N}^{-1}(\theta) \bar{g}(\theta),
\end{aligned}
$$

where, as before,

$$
W_{N}(\theta)=\frac{1}{N} \sum_{i=1}^{N} g_{i}(\theta) g_{i}(\theta)^{\prime}
$$

and so the CUE minimises the criterion function including the parameters in the weight matrix. The limiting distribution under standard regularity conditions is given by

$$
\sqrt{N}\left(\widehat{\theta}_{C U}-\theta_{0}\right) \rightarrow N(0, V) ; \quad V=\left(\Gamma_{\theta_{0}}^{\prime} \Psi^{-1} \Gamma_{\theta_{0}}\right)^{-1}
$$

and is the same as the efficient two-step GMM estimator. The asymptotic variance of the CUE is computed as

$$
\widehat{\operatorname{var}}\left(\widehat{\theta}_{C U}\right)=\frac{1}{N}\left(C\left(\widehat{\theta}_{C U}\right)^{\prime} W_{N}^{-1}\left(\widehat{\theta}_{C U}\right) C\left(\widehat{\theta}_{C U}\right)\right)^{-1}
$$

which is used in the calculation of the standard Wald test. Again, it has been shown by e.g. Hansen, Heaton and Yaron (1996) that the asymptotic standard errors are severely downward biased, leading to overrejection of a true null hypothesis using the Wald test.

Newey and Windmeijer (2005) derive the asymptotic distribution of the CUE under many weak instrument asymptotics. In these asymptotics, the number of instruments is allowed to grow with the sample size $N$, with the increase in number of instruments accompanied by an increase in the concentration parameter. The resulting limiting distribution of the CUE is again the normal distribution, but convergence is at a slower rate than $\sqrt{N}$. The asymptotic variance is in this case larger than the asymptotic variance using conventional asymptotics, and can be estimated consistently by

$$
\widehat{\operatorname{var}}\left(\widehat{\theta}_{C U}\right)_{c}=\frac{1}{N} H^{-1}\left(\widehat{\theta}_{C U}\right) S\left(\widehat{\theta}_{C U}\right)^{\prime} W_{N}^{-1}\left(\widehat{\theta}_{C U}\right) S\left(\widehat{\theta}_{C U}\right) H^{-1}\left(\widehat{\theta}_{C U}\right),
$$


where

$$
\begin{aligned}
H(\theta) & =\frac{\partial^{2} Q(\theta)}{\partial \theta \partial \theta^{\prime}} ; \quad S(\theta)=\left(S_{1}(\theta), S_{2}(\theta), \ldots, S_{k}(\theta)\right) \\
S_{j}(\theta) & =\left(\frac{\partial \bar{g}(\theta)}{\partial \theta_{j}}-\Lambda_{j}(\theta) W_{N}^{-1}(\theta) \bar{g}(\theta)\right) \\
\Lambda_{j}(\theta) & =\frac{1}{N} \sum_{i=1}^{N} \frac{\partial g_{i}(\theta)}{\partial \theta_{j}} g_{i}(\theta)^{\prime} .
\end{aligned}
$$

Here, unlike the usual asymptotics, the middle matrix $S\left(\widehat{\theta}_{C U}\right)^{\prime} W_{N}^{-1}\left(\widehat{\theta}_{C U}\right) S\left(\widehat{\theta}_{C U}\right)$ estimates a different, larger object than the Hessian. Also, the use of the Hessian is important, as the more common formula $C\left(\widehat{\theta}_{C U}\right)^{\prime} W_{N}^{-1}\left(\widehat{\theta}_{C U}\right) C\left(\widehat{\theta}_{C U}\right)$ has extra random terms that are eliminated in the Hessian under the alternative asymptotics.

Hansen, Heaton and Yaron (1996) proposed the use of a criterion-based test similar to $D_{R U}$, but based on the CUE. Their test statistic $D_{R U}^{C U}$ is defined as

$$
D_{R U}^{C U}=2 N\left(Q\left(\widetilde{\theta}_{C U}\right)-Q\left(\widehat{\theta}_{C U}\right)\right)
$$

where $\widehat{\theta}_{C U}$ and $\widetilde{\theta}_{C U}$ are the CUEs for the unrestricted and restricted models respectively. Under the null, $D_{R U}^{C U}$ has an asymptotic $\chi_{r}^{2}$ distribution.

\subsection{Monte Carlo results}

In this section we will illustrate the finite sample performance of the GMM estimators and the test statistics as discussed in the previous sections by means of a small Monte Carlo study. The data generating process is given by

$$
\begin{aligned}
y_{i t} & \sim \text { Poisson }\left(\exp \left(x_{i t} \beta+\eta_{i}+\varepsilon_{i t}\right)\right) \\
x_{i t} & =\rho x_{i t-1}+\delta \eta_{i}+\theta \varepsilon_{i t-1}+\omega_{i t} \\
\eta_{i} & \sim N\left(0, \sigma_{\eta}^{2}\right) ; \varepsilon_{i t} \sim N\left(0, \sigma_{\varepsilon}^{2}\right) ; \omega_{i t} \sim N\left(0, \sigma_{\omega}^{2}\right), \\
\beta & =0.5 ; \delta=0.1 ; \theta=0.3 ; \sigma_{\eta}^{2}=0.3 ; \sigma_{\varepsilon}^{2}=0.3 ; \sigma_{\omega}^{2}=0.25 \\
\rho & =\{0.5,0.8\} .
\end{aligned}
$$

The dependent variable is a count variable, generated from the Poisson distribution with unobserved fixed normally distributed heterogeneity $\eta_{i}$ and further idiosyncratic normally 
distributed heterogeneity $\varepsilon_{i t}$. The $x_{i t}$ are correlated with the $\eta_{i}$ and $\varepsilon_{i t-1}$ and are therefore predetermined.

Table 1 presents estimation results from 10, 000 Monte Carlo replications for the oneand two-step GMM estimators as well as the continuous updating estimator for $T=6$, $N=250$ and $\rho=0.5$ or $\rho=0.8$, using the moment conditions (6) as proposed by Chamberlain (1992). The instruments set is given by

$$
Z_{i}=\left[\begin{array}{lllllll}
x_{i 1} & & & & & & \\
& x_{i 1} & x_{i 2} & & & & \\
& & & \ddots & & & \\
& & & & x_{i 1} & \cdots & x_{i T-1}
\end{array}\right]
$$

and hence there are a total of 15 moment conditions.The one-step GMM estimator uses $W_{N}=\frac{1}{N} \sum_{i=1}^{N} Z_{i}^{\prime} Z_{i}$ as the weight matrix.

When $\rho=0.5$, the instruments are quite strong. The one-step GMM estimator, denoted GMM1 in the table, has a moderate downward bias of -0.0408. Its standard deviation is 0.1053 , which seems well approximated by the asymptotic standard error. The mean of the estimated standard errors is equal to 0.1031. The two-step GMM estimator, denoted GMM2, has a smaller bias of -0.0211 and a smaller standard deviation of 0.0803 , representing a substantial efficiency gain with more than a $23 \%$ reduction in standard deviation. In contrast to the one-step estimator, the mean of the usual asymptotic standard errors is $19 \%$ smaller than the standard deviation. However, taking account of the extra variation due to the presence of the one-step estimates in the weight matrix results in finite sample corrected standard errors with a mean of 0.0799 , which is virtually identical to the standard deviation. The CUE has a very small bias of 0.0043 , with a standard deviation of 0.0904, which is larger than that of the two-step GMM estimator, but smaller than that of the one-step estimator. The mean of the usual asymptotic standard errors is exactly the same as that of the two-step GMM estimator and in this case it is almost $28 \%$ smaller than the standard deviation. The standard errors resulting from the many weak instruments asymptotics have a mean of 0.0918, which is virtually the same as the standard deviation. 
Table 1. Estimation results

\begin{tabular}{lrcccrc}
\hline \hline$\rho=0.5$ & mean bias & st dev & se & sec & median bias & IQR \\
GMM1 & -0.0408 & 0.1053 & 0.1031 & & -0.0409 & 0.1381 \\
GMM2 & -0.0211 & 0.0803 & 0.0652 & 0.0799 & -0.0209 & 0.1077 \\
CUE & 0.0043 & 0.0904 & 0.0652 & 0.0918 & 0.0024 & 0.1165 \\
\hline$\rho=0.8$ & & & & & & \\
GMM1 & -0.1136 & 0.2094 & 0.1773 & & -0.0974 & 0.2435 \\
GMM2 & -0.0537 & 0.1335 & 0.0908 & 0.1365 & -0.0498 & 0.1558 \\
CUE & 0.0033 & 0.1885 & 0.0879 & 0.1459 & 0.0029 & 0.1742 \\
\hline \hline
\end{tabular}

Note: $T=6, N=250,10000$ replications, sec denotes finite sample corrected standard errors for GMM2 and those resulting from many weak instrument asymptotics for CUE, IQR=Inter Quartile Range

Figure 1 shows p-value plots for the hypothesis $H_{0}: \rho=0.5$, comparing nominal size with rejection frequencies. The various Wald tests are denoted $W_{1}, W_{2}, W_{2 C}, W_{C U}$ and $W_{C U C}$ based on one-step GMM, two-step GMM with usual standard errors, two-step GMM with finite sample corrected standard errors, CUE and CUE with standard errors resulting from the many weak instruments asymptotics, respectively. As expected, $W_{2}$ and $W_{C U}$ overreject the null hypothesis substantially. $W_{1}$ and $W_{2 C}$ perform much better, but are still moderately oversized due to the bias of the estimators. $W_{C U C}$ has a very good performance in terms of size of the test, the rejection frequencies being very close to the $45^{\circ}$ line. The two-step GMM based LM and $D_{R U}$ tests also perform very well, their p-value plots being quite similar to that of $W_{C U C}$. Finally, the CUE based $D_{R U}^{C U}$ performs well, but tends to overreject moderately at higher values of the nominal size.

When $\rho=0.8$, the instruments become weaker as the $x_{i t}$ series become more persistent. The one-step GMM estimator now has a quite large downward bias of -0.1136 . Its standard deviation is 0.2094 , which is now less well approximated by the asymptotic standard error, with the mean of the estimated standard errors being equal to 0.1773 . The two-step GMM estimator has a smaller, but still quite substantial bias of -0.0537 and a smaller standard deviation of 0.1335 . The mean of the usual asymptotic standard errors is 0.0908 , again substantially smaller than the standard deviation. The mean of the finite sample corrected standard errors is 0.1365 , which is again very close to the standard deviation. The CUE, also with these weaker instruments, has a very small bias 


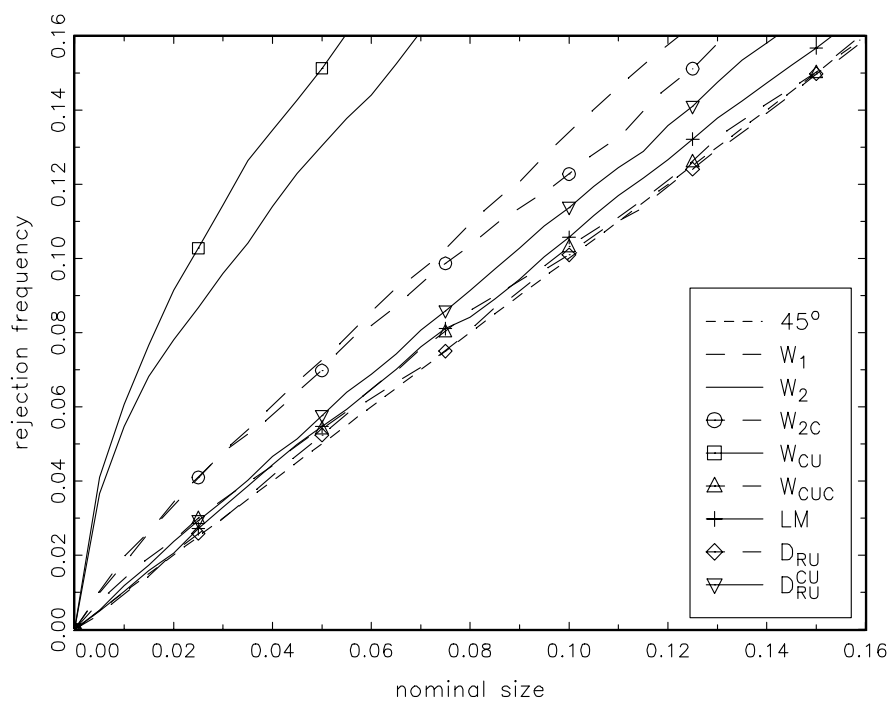

Figure 1. P-value plot, $H_{0}: \rho=0.5$

of 0.0033 , with a standard deviation of 0.1885 . In this case the so-called no momentproblem starts to become an issue for the CUE, though, with some outlying estimates inflating the standard deviation, see Guggenberger (2005). It is therefore better to look at the median bias and inter quartile range (IQR) in this case, which shows that the CUE is median unbiased with an IQR which is only slightly larger than that of the two-step GMM estimator, 0.1742 versus 0.1558 respectively.

Figure 2 shows p-value plots for the hypothesis $H_{0}: \rho=0.8 . W_{2}$ and $W_{C U}$ overreject the null hypothesis even more than when $\rho=0.5$. $W_{1}$ performs better, but is still substantially oversized. $W_{2 C}$ and $W_{C U C}$ perform quite well and quite similar, slightly overrejecting the null. The two-step GMM based LM and $D_{R U}$ are again the best performers in terms of size, whereas the CUE based $D_{R U}^{C U}$ performs worse than $W_{2 C}$ and $W_{C U C}$. 


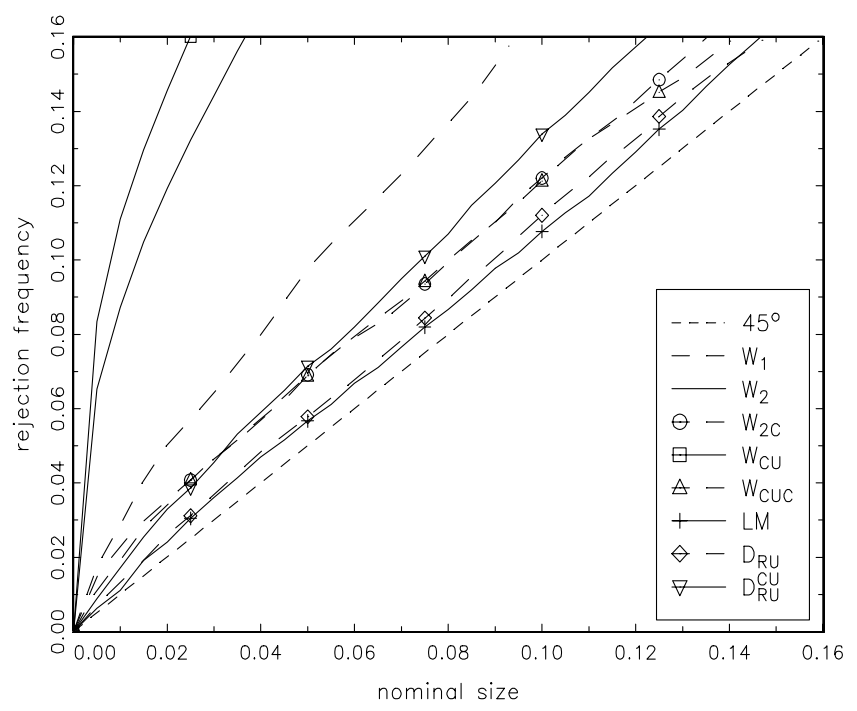

Figure 2. P-value plot, $H_{0}: \rho=0.8$

Summarising, it is clear that use of the finite sample corrected standard errors for the two-step GMM estimator and the standard errors from the many weak instrument asymptotics for the CUE improve the size performance of the Wald tests for these estimators considerably. The simple criterion based $D_{R U}$ test performs very well in these examples, as was the case in Bond and Windmeijer (2005) for linear panel data models.

\section{References}

Angrist J.D., 2001, Estimation of limited dependent variable models with dummy endogenous regressors: simple strategies for empirical practice, Journal of Business \& Economic Statistics 19, 2-16.

Aptech Systems, Inc., 2005, GAUSS, Advanced Mathematical and Statistical System, Black Diamond, WA, USA.

Blundell R., R. Griffith and J. van Reenen, 1999, Market share, market value and innovation in a panel of British manufacturing firms, Review of Economic Studies $66,529-554$. 
Blundell R., R. Griffith and F. Windmeijer, 2002, Individual effects and dynamics in count data models, Journal of Econometrics 108, 113-131.

Bond S.R., C. Bowsher and F. Windmeijer, 2001, Criterion-Based inference for GMM in autoregressive panel data models, Economics Letters 73, 379-388.

Bond S.R. and F. Windmeijer, 2005, Reliable inference for GMM estimators? Finite sample procedures in linear panel data models, Econometric Reviews 24, 1-37.

Chamberlain G., 1992, Comment: Sequential moment restrictions in panel data, Journal of Business \& Economic Statistics 10, 20-26.

Cincera M., 1997, Patents, R\&D, and technological spillovers at the firm level: some evidence from econometric count models for panel data, Journal of Applied Econometrics $12,265-280$.

Crépon B. and E. Duguet, 1997, Estimating the innovation function from patent numbers: GMM on count panel data, Journal of Applied Econometrics 12, 243-263.

Greene, W.H., 2005, LIMDEP 8.0, Econometric Software, Inc., Plainview, NY, USA.

Guggenberger P., 2005, Monte-carlo evidence suggesting a no moment problem of the continuous updating estimator, Economics Bulletin 3, 1-6.

Hall B. and C. Cummins, 2005, TSP 5.0, TSP International, Palo Alto, CA, USA.

Hansen L.P., 1982, Large sample properties of generalized method of moments estimators, Econometrica 50, 1029-1054.

Hansen L.P., J. Heaton and A. Yaron, 1996, Finite-sample properties of some alternative GMM estimators, Journal of Business \& Economic Statistics 14, 262-280.

Hausman J., B. Hall and Z. Griliches, 1984, Econometric models for count data and an application to the patents-R\&D relationship, Econometrica 52, 909-938. 
Kelly M., 2000, Inequality and crime, The Review of Economics and Statistics 82, 530539.

Kitazawa Y., 2000, TSP procedures for count panel data estimation, Kyushu Sangyo University.

Lancaster T., 2002, Orthogonal parameters and panel data, Review of Economic Studies 69, 647-666.

Kim J. and G. Marschke, 2005, Labor mobility of scientists, technological diffusion and the firm's patenting decision, The RAND Journal of Economics 36, 298-317.

Manning W.G., A. Basu and J. Mullahy, 2005, Generalized modeling approaches to risk adjustment of skewed outcomes data, Journal of Health Economics 24, 465-488.

Montalvo J.G., 1997, GMM estimation of count-panel-data models with fixed effects and predetermined instruments, Journal of Business and Economic Statistics 15, $82-89$.

Mullahy J., 1997, Instrumental variable estimation of Poisson regression models: application to models of cigarette smoking behavior, Review of Economics and Statistics $79,586-593$.

Newey W.K. and R.J. Smith, 2004, Higher order properties of GMM and generalized empirical likelihood estimators, Econometrica 72, 219-255.

Newey W.K. and K.D. West, 1987, Hypothesis testing with efficient method of moments estimation, International Economic Review 28, 777-787.

Newey W.K. and F. Windmeijer, 2005, GMM with many weak moment conditions, cemmap Working Paper No CWP18/05.

Romeu A., 2004, ExpEnd: Gauss code for panel count data models, Journal of Applied Econometrics 19, 429-434. 
Salomon R.M. and J.M. Shaver, 2005, Learning by exporting: new insights from examining firm innovation, Journal of Economics and Management Strategy 14, 431-460.

Santos Silva J.M.C. and S. Tenreyro, 2006, The log of gravity, The Review of Economics and Statistics 88.

Schellhorn M., 2001, The effect of variable health insurance deductibles on the demand for physician visits, Health Economics 10, 441-456.

Vera-Hernandez, A.M., 1999, Duplicate coverage and demand for health care. The case of Catalonia, Health Economics 8, 579-598.

Windmeijer F., 2000, Moment conditions for fixed effects count data models with endogenous regressors, Economics Letters 68, 21-24.

Windmeijer F., 2002, ExpEnd, a Gauss programme for non-linear GMM estimation of exponential models with endogenous regressors for cross section and panel data, cemmap Working Paper No. CWP14/02.

Windmeijer F., 2005, A finite sample correction for the variance of linear efficient twostep GMM estimators, Journal of Econometrics 126, 25-517.

Windmeijer F. and J.M.C. Santos Silva, 1997, Endogeneity in count data models: an application to demand for health care, Journal of Applied Econometrics 12, 281294.

Wooldridge J.M., 1991, Multiplicative panel data models without the strict exogeneity assumption, MIT Working Paper No. 574.

Wooldridge J.M., 1997, Multiplicative panel data models without the strict exogeneity assumption, Econometric Theory 13, 667-678.

Wooldridge J.M., 1999, Distribution-free estimation of some nonlinear panel data models, Journal of Econometrics 90, 77-97. 